IFT $/ 4 / 99$

\title{
Boost-Invariant Running Couplings in Effective Hamiltonians
}

\author{
May, 1999 \\ Stanisław D. Głazek \\ Institute of Theoretical Physics, Warsaw University \\ ul. Hoża 69, 00-681 Warsaw
}

\begin{abstract}
We apply a boost-invariant similarity renormalization group procedure to a light-front Hamiltonian of a scalar field $\phi$ of bare mass $\mu$ and interaction term $\sim g \phi^{3}$ in 6 dimensions using 3rd order perturbative expansion in powers of the coupling constant $g$. The initial Hamiltonian is regulated using momentum dependent factors that approach 1 when a cutoff parameter $\Delta$ tends to infinity. The similarity flow of corresponding effective Hamiltonians is integrated analytically and two counterterms depending on $\Delta$ are obtained in the initial Hamiltonian: a change in $\mu$ and a change of $g$. In addition, the interaction vertex requires a $\Delta$-independent counterterm that contains a boost invariant function of momenta of particles participating in the interaction. The resulting effective Hamiltonians contain a running coupling constant that exhibits asymptotic freedom. The evolution of the coupling with changing width of effective Hamiltonians agrees with results obtained using Feynman diagrams and dimensional regularization when one identifies the renormalization scale with the width. The effective light-front Schrödinger equation is equally valid in a whole class of moving frames of reference including the infinite momentum frame. Therefore, the calculation described here provides an interesting pattern one can attempt to follow in the case of Hamiltonians applicable in particle physics.
\end{abstract}

PACS Numbers: 11.10.Gh 


\section{INTRODUCTION}

Similarity renormalization group procedure for Hamiltonians is a method suggested for seeking solutions to QCD and other quantum field theories en bloc including bound states, by calculating manageable effective Hamiltonians and solving Schrödinger equations with them in a series of successive approximations of increasing accuracy. This article describes an elementary application of the similarity method in case of scalar fields, showing details of a calculation of one boost-invariant running coupling constant in effective Hamiltonians in third order perturbation theory. We briefly review the method, describe the simple example and present conclusions.

The method originates in the notion of renormalization group as discussed by Wilson [1] [2] and uses the idea of similarity renormalization group procedure for Hamiltonians [3] [ [4]. Similarity enables us to avoid small energy denominators in perturbative evaluation of effective Hamiltonians. The evaluation includes finding counterterms and defining renormalized dynamics. Small energy denominators could lead to large errors in the counterterms and in calculation of effective Hamiltonians. Thus, if not avoided through similarity, the small denominators would prevent precise theoretical predictions based on the effective Schrödinger equations.

The similarity procedure was invented to sort out complexities of the light-front form of Hamiltonian dynamics. This form was distinguished a long time ago by Dirac [5] and more recently became a natural candidate for description of hadronic constituents in hard scattering processes [6] as well as in spectroscopy [7] using QCD. A recent review article by Brodsky, Pauli and Pinsky [8] provides a description of theoretical advances made in light-front formulation of various theories mainly before invention or independently of the similarity procedure. Reviews by Burkardt, Harindranath and Perry [9] help in understanding the scope of current approaches. Recent research in the direction of renormalization of Hamiltonians can be traced through Ref. [10]. The present article is focused on similarity in light-front dynamics.

Initial studies of quarkonium bound states, which are related to the similarity program described in [7] have been performed by Brisudová and Perry, and Brisudová, Perry and Wilson [11] following the key observation by Perry [12] that second order effective Hamiltonian of QCD contains a confining term, which may remain uncanceled in the effective Schrödinger equation dynamics. Higher than second order calculations are needed for verifying this hypothesis.

Since the formal front form of Hamiltonian dynamics is invariant under boosts one hopes it can provide a link between the structure of hadrons at rest and in the parton model. That such unifying picture is hard to achieve in standard dynamical schemes is best illustrated by the fact that despite extensive progress lattice gauge theory does not easily yield desired quark and gluon bound state wave functions. [13 The light-front approach is still far from achieving this goal, too. The present article shows the essence of boost invariance in similarity [14] but the example we describe here is limited to scalar particles. The dynamics of scalars does not involve genuine small- $x$ singularities $(x$ denotes a longitudinal momentum fraction carried by a particle in the infinite momentum frame) that appear in gauge theories and the present work does not describe known particles.

The present paper also does not cover the step of solving the effective Schrödinger equation. It was shown in an asymptotically free matrix model [15] that one can achieve $10 \%$ accuracy in calculating bound state properties using second order effective Hamiltonians but it is not known yet if the same accuracy can be reached in any quantum field theory of interest using 
the boost-invariant similarity approach discussed here.

Before we proceed to our example, we first disclose the mechanism of preserving boost invariance in the similarity approach. The following discussion points out relevant features of the procedure using other methods for comparison.

The similarity renormalization group procedure leads to effective Hamiltonians $H_{\lambda}=H_{0 \lambda}+$ $H_{I \lambda}$, whose matrix elements between any two eigenstates of $H_{0 \lambda}$ vanish when the eigenvalues of $H_{0 \lambda}$ for these states differ by more than the "width" $\lambda$. The word "width" is natural because the effective Hamiltonian matrix can be viewed as a band of non-zero matrix elements along the diagonal and the width of the band depends on $\lambda$. Another reason for the word "width" is that the band structure is ensured by similarity form factors in interaction vertices. The form factors are functions of energy transfers, they are peaked around zero and their half-width depends on $\lambda$. The notion of the Hamiltonian width is key to our method of preserving boost invariance.

We shall take advantage of Wegner's equation [16 to indicate where the boost invariance can enter the similarity procedure. Wegner invented a flow equation for diagonalization of Hamiltonian matrices in solid state physics [16] [17 that is beautifully simple and can be adapted to the similarity renormalization scheme [18] [15]. Wegner's equation for Hamiltonian matrices can be written as

$$
\frac{d}{d s} H_{\lambda}=-\left[\left[H_{\lambda}, H_{0 \lambda}\right], H_{\lambda}\right]
$$

where $s=\lambda^{-2}$. Initial condition should be provided at $s=0$, corresponding to $\lambda=\infty$, and the initial Hamiltonian is denoted by $H_{\infty}$. Wegner's commutator $\left[H_{\lambda}, H_{0 \lambda}\right]$ generates the similarity transformation. One discovers a gaussian similarity factor by solving Eq. (1.1) for the $H_{I \lambda}$ matrix elements between eigenstates of matrix $H_{0 \lambda}$ keeping on the right-hand side only those terms that are linear in $H_{I \lambda}$ and neglecting higher order terms.

Wegner's equation preserves necessary cluster decomposition properties [19]. The commutator structure of Wegner's equation implies that no disconnected interactions are generated by the transformation. Wegner's transformation depends only on differences of energies and spectator energies always drop out from the differences. However, Wegner's generator is not boost invariant.

Seeking a boost invariant approach, one can apply Eq. (1.1) to light-front Hamiltonian matrices. The matrices are of the form $H=\left(P^{\perp 2}+M_{\lambda}^{2}\right) / P^{+}$, where the mass matrix contains interactions, $M_{\lambda}^{2}=M_{0 \lambda}^{2}+M_{I \lambda}^{2}$, and $P$ denotes total momenta of states with which one evaluates the matrix elements. Since the masses do not change the momenta, one can rewrite Eq. (1.1) in terms of the mass matrix elements as follows

$$
P^{+2} \frac{d}{d s} M_{\lambda}^{2}=-\left[\left[M_{\lambda}^{2}, M_{0 \lambda}^{2}\right], M_{\lambda}^{2}\right]
$$

We see that a rescaling of the flow parameter $s$ with a momentum eigenvalue gives a flow equation for the mass squared matrix elements. The latter should be independent of the eigenvalues $P^{+}$and $P^{\perp}$. This feature of masses seems to suggest a boost invariant renormalization procedure based on replacing $P^{+2} / s$ by a new $P^{+}$-independent flow parameter $\Lambda^{4}$. But such substitution breaks connection with Wegner's equation for Hamiltonian matrices and leads to violation of cluster decomposition properties, since the mass is not an additive quantity. 
Namely, the mass depends on the relative motion of particles and effective interactions become dependent on spectators momentum.

Despite this drawback, Allen and Perry [20] succeeded in demonstration that one can define and calculate a running coupling constant using Eq. (1.2) for $M^{2}$ matrix elements replacing $P^{+2} / s$ with $\Lambda^{4}$ in massless $\phi^{3}$ theory in 6 dimensions. The authors demand that the flow from some $\Lambda$ to a fraction of $\Lambda$ reproduces the same matrix elements structure in which only some parameters change. This condition is implemented using transverse locality and it allows for bypassing the step of finding initial conditions (counterterms) needed for differential equations, by introducing a running coupling constant. A question arises because studies of asymptotically free matrix models [15] [21] show that effective Hamiltonian matrices that are suitable for bound state calculations may significantly deviate from a self-replicating (fixed point) flow with one coupling constant. But in the case of many couplings helpful conditions may be provided by coupling coherence [11, which may work in the approach of Eq. (1.2) with a $P^{+}$ independent width parameter, assuming that the cluster property breaking does not complicate the coherence. Kylin, Allen and Perry [22] extended the approach of Allen and Perry to massive particles.

The similarity renormalization group procedure for Hamiltonians is more flexible in defining its generator than Eqs. (1.1) and (1.2) might suggest. This flexibility is used to preserve boost symmetry and cluster properties simultaneously. Note that the boost invariance we talk about means invariance under boosts along the front and boosts corresponding to rotations about transverse axes in the infinite momentum frame. These boosts are sufficient for reaching states in all possible uniform motions.

The desired boost-invariant operator formulation of similarity [14] is based on a transformation $\mathcal{U}_{\lambda}$ that changes creation and annihilation operators of bare particles (denoted by $q_{\infty}$, since they correspond to $\lambda=\infty$ ) into creation and annihilation operators of effective particles corresponding to the width $\lambda$ (denoted by $q_{\lambda}$ ). Namely,

$$
q_{\lambda}=\mathcal{U}_{\lambda} q_{\infty} \mathcal{U}_{\lambda}^{\dagger}
$$

$\mathcal{U}_{\lambda}$ is secured to be unitary by construction. Hamiltonian operators of all widths are assumed equal and when they are expressed in terms of different creation and annihilation operators, the coefficient functions change. We have $H_{\lambda}\left(q_{\lambda}\right)=H_{\infty}\left(q_{\infty}\right)$. Assuming that Hamiltonians calculable in perturbation theory contain only finite products of creation and annihilation operators and applying the transformation $\mathcal{U}_{\lambda}$, one obtains $\mathcal{H}_{\lambda} \equiv H_{\lambda}\left(q_{\infty}\right)=\mathcal{U}_{\lambda}^{\dagger} H_{\infty}\left(q_{\infty}\right) \mathcal{U}_{\lambda}$. This relation means that the operator $\mathcal{H}_{\lambda}$ has the same coefficient functions in front of products of $q_{\infty}$ as the effective Hamiltonian $H_{\lambda}$ has in front of the unitarily equivalent products of $q_{\lambda}$. Differentiating $\mathcal{H}_{\lambda}$ one obtains

$$
\frac{d}{d \lambda} \mathcal{H}_{\lambda}=-\left[\mathcal{T}_{\lambda}, \mathcal{H}_{\lambda}\right]
$$

where the generator $\mathcal{T}_{\lambda}$ is related to $\mathcal{U}_{\lambda}$ by

$$
\mathcal{T}_{\lambda}=\mathcal{U}_{\lambda}^{\dagger} \frac{d}{d \lambda} \mathcal{U}_{\lambda}
$$

The script letters are introduced in Eqs. (1.3a-c) to indicate that the operators can be conveniently thought about as expanded into sums of products of operators $q_{\infty}$. The latter are 
independent of $\lambda$ and are not differentiated in Eqs. (1.3b-c). In other words, Eqs. (1.3b-c) describe only the flow of coefficients in front of the creation and annihilation operators. Effective Hamiltonians are obtained from $\mathcal{H}_{\lambda}$ using $H_{\lambda}\left(q_{\lambda}\right)=\mathcal{U}_{\lambda} \mathcal{H}_{\lambda} \mathcal{U}_{\lambda}^{\dagger}$.

Note that the operator $\mathcal{U}_{\lambda}$ is analogous to the Melosh operator [23]. The analogy stems from that the effective particles corresponding to a small Hamiltonian width $\lambda$ can be associated with constituent quarks or gluons from hadronic rest frame spectroscopy while the bare particles with $\lambda \rightarrow \infty$ can be associated with current quarks and gluons from the infinite momentum frame. The boost-invariant similarity renormalization group procedure for light-front Hamiltonians 14. makes the notions of current and constituent quarks and gluons boost invariant. The procedure provides a calculable dynamical content to the transformation that connects the current and constituent particles independently of the reference frame one works in. This may sound confusing if Melosh transformation is associated with changing a frame of reference. But the light-front dynamics is invariant with respect to boosts in perturbative calculations and this property allows for translating a Melosh-like transformation to one frame of reference, where it simply relates bare particles and complex effective particles in one theory in the same frame.

Boost invariance is guaranteed through the definition of the generator $\mathcal{T}_{\lambda}$. Details will be reviewed in the next Section. The effective Hamiltonian $H_{\lambda}$ is given by a diagonal proximum of certain operator $G_{\lambda}$, i.e. $H_{\lambda}=F_{\lambda}\left[G_{\lambda}\right]$, while the generator $\mathcal{T}_{\lambda}$ is related to a diagonal remotum of the same operator $G_{\lambda}$. Following [14], we introduce $\mathcal{G}_{\lambda}=\mathcal{U}_{\lambda}^{\dagger} G_{\lambda} \mathcal{U}_{\lambda}$ and use

$$
\left[\mathcal{T}_{\lambda}, \mathcal{H}_{0 \lambda}\right]=\frac{d}{d \lambda}\left(1-F_{\lambda}\right)\left[\mathcal{G}_{\lambda}\right]
$$

The commutator structure guarantees that effective Hamiltonians contain only connected interactions.

Now, the diagonal proximum can be defined in a boost-invariant way. The point is that the operation $F_{\lambda}$ multiplies every interaction term in an effective Hamiltonian by a form factor which is a function of boost invariant combinations of momenta labeling creation and annihilation operators in that interaction term. Such combinations can include differences or sums of invariant masses. The resulting generator of the similarity transformation does not depend on the total momentum of any state, contrary to Eq. (1.2). According to [14], a necessary rescaling of the flow parameter, analogous to rescaling provided by the total $P^{+}$in Eq. (1.2), is provided for each Hamiltonian term separately using a parent three-momentum for that term. The parent momentum is defined as half of the sum of all momenta labeling creation and annihilation operators in the term in question. All light-front Hamiltonians we consider are sums of terms which contain integrals over the parent momenta. Usually, when a term acts on some state, a parent momentum equals only some fraction of the total momentum of that state. This fraction is defined by the single interaction act and lies in a range of values allowed by momentum conservation in the effective Schrödinger dynamics. Thus, effective interactions are independent of the total momenta of states they act on and no dependence on spectators is generated. Therefore, no cluster property is violated in defining the similarity generator through Eq. (1.4). A considerable freedom is still left in choosing details of $F_{\lambda}$.

In summary, the flexibility available in defining the similarity transformation generator can be used to obtain a boost-invariant band-diagonal structure of effective Hamiltonians preserving cluster decomposition properties, through a suitable choice of the similarity form factors $F_{\lambda}$. This aspect of the procedure is the subject of the present article, quite independently of which 
theory or singularity is addressed. Naturally, the effective theory is easiest to make boost invariant if the initial Hamiltonian is regulated in a boost invariant way. For in that case the counterterms do not have to correct the boost invariance breaking which a frame dependent regularization would introduce. A suitable class of regularizations will be described in the next Section.

This paper is organized as follows. Section 2 reviews necessary elements of Ref. [14] set up for a third order calculation of the running coupling constant in Hamiltonians of scalar $\phi^{3}$ theory in 6 dimensions. The calculation is based on a plain power series expansion in the coupling constant. Counterterms are derived in Section 3 and the effective coupling constant flow is calculated in Section 4. Section 5 concludes the paper. Appendix presents formulae for third order effective vertex function with arbitrary momenta and masses.

\section{EFFECTIVE HAMILTONIANS}

We focus our attention on a perturbative derivation of effective Hamiltonians in scalar quantum field theory with an interaction term $\sim \phi^{3}$ in 6 dimensions. The theory is known to be asymptotically free. The coupling constant in the initial regularized Hamiltonian is assumed to be infinitesimally small. Our procedure for calculating effective Hamiltonians closely follows Ref. [14].

\subsection{Initial Hamiltonian}

The classical Lagrangian of the scalar field theory in question is

$$
\mathcal{L}=\frac{1}{2}\left(\partial_{\mu} \phi \partial^{\mu} \phi-\mu^{2} \phi^{2}\right)-\frac{g}{3 !} \phi^{3}
$$

The corresponding light-front Hamiltonian reads [24] 25] $\left(x^{ \pm}=x^{0} \pm x^{3}\right)$

$$
H=\int d x^{-} d^{4} x^{\perp}\left[\frac{1}{2} \phi\left(-\partial^{\perp 2}+\mu^{2}\right) \phi+\frac{g}{3 !} \phi^{3}\right]_{x^{+}=0} .
$$

A quantum field $\phi(x)$ for $x^{+}=0$ is expanded in its Fourier components,

$$
\left.\phi(x)\right|_{x^{+}=0}=\left.\int_{\delta^{+}}[k]\left(a_{k} e^{-i k x}+a_{k}^{\dagger} e^{i k x}\right)\right|_{x^{+}=0},
$$

where the abbreviated notation for the integral means

$$
\int_{\delta^{+}}[k]=\int_{\delta^{+}}^{\infty} \frac{d k^{+}}{2 k^{+}} \int \frac{d^{4} k^{\perp}}{(2 \pi)^{5}} .
$$

The small parameter $\delta^{+}$limits the longitudinal momenta, $k^{+}$, from below. The creation and annihilation operators satisfy commutation relations

$$
\left[a_{k}, a_{q}^{\dagger}\right]=2 k^{+}(2 \pi)^{5} \delta\left(k^{+}-q^{+}\right) \delta^{4}\left(k^{\perp}-q^{\perp}\right)
$$

together with $\left[a_{k}, a_{q}\right]=\left[a_{k}^{\dagger}, a_{q}^{\dagger}\right]=0$. Substituting Eq. (2.3) in (2.2) one obtains the following unique structure of the light-front Hamiltonian 


$$
H=\int_{\delta^{+}}[k] \frac{k^{\perp 2}+\mu^{2}}{k^{+}} a_{k}^{\dagger} a_{k}+\frac{g}{2} \int_{\delta^{+}}[k p q] 2(2 \pi)^{5} \delta^{5}(k+p-q)\left(a_{k}^{\dagger} a_{p}^{\dagger} a_{q}+a_{q}^{\dagger} a_{p} a_{k}\right)
$$

It is unique in comparison to Hamiltonians in other forms of dynamics because there are no terms that contain only creation or only annihilation operators in Eq. (2.6). This feature is related to the problem of ground state formation, since $H|0\rangle=0$, where $a_{k}|0\rangle=0$, and only $a_{0}^{\dagger} a_{0}^{\dagger} a_{0}^{\dagger}$ and $a_{0}^{\dagger}$ terms in $H$ could alter this feature, but they are absent due to the cutoff $\delta^{+}$. Readers interested in non-perturbative aspects of Hamiltonian dynamics in the front form should consult [26]. However, no problems with modes of $k^{+}=0$ arise in perturbation theory for Hamiltonians in the present paper.

The initial Hamiltonian for the similarity renormalization group procedure is obtained from Eq. (2.6) by introducing an additional ultraviolet regularization factor (to be described below) and taking the limit $\delta^{+} \rightarrow 0$. The initial Hamiltonian is denoted by $H_{\infty}$, since it corresponds to the initial width $\lambda=\infty$ in the similarity flow, as discussed in the Introduction. Creation and annihilation operators that appear in $H_{\infty}$ were denoted in the Introduction by $q_{\infty}$.

$H_{\infty}$ contains a regularization factor denoted by $r_{\Delta} . \Delta$ stands for an ultraviolet cutoff parameter. $r_{\Delta} \rightarrow 1$ when $\Delta \rightarrow \infty$. In asymptotically free theories, $\Delta$ can be sent to infinity when the renormalization process passes the stage of calculating counterterms and deriving effective Hamiltonians of finite width $\lambda$. Still, the initial Hamiltonian contains counterterms which depend on $r_{\Delta}$ and are denoted by $X_{\Delta}$. Besides diverging $\Delta$-dependent terms, $X_{\Delta}$ contains also finite parts that remove $\Delta$-independent regularization effects caused by the factor $r_{\Delta}$. Thus, we have

$$
\begin{gathered}
H_{\infty}=\int[k] \frac{k^{\perp 2}+\mu^{2}}{k^{+}} a_{\infty k}^{\dagger} a_{\infty k}+ \\
+\frac{g}{2} \int\left[k_{1} k_{2} k_{3}\right] 2(2 \pi)^{5} \delta^{5}\left(k_{1}+k_{2}-k_{3}\right)\left(a_{\infty k_{1}}^{\dagger} a_{\infty k_{2}}^{\dagger} a_{\infty k_{3}}+a_{\infty k_{3}}^{\dagger} a_{\infty k_{2}} a_{\infty k_{1}}\right) r_{\Delta}+X_{\Delta} .
\end{gathered}
$$

The parameter $\delta^{+}$is set equal 0 and this is why it is not indicated, in distinction from Eq. (2.6) where it was kept larger than 0 .

The regularization factor $r_{\Delta}$ has a simple form which results from the following steps (applicable to the interaction term written out explicitly in Eq. (2.7) as well as to all counterterms $X_{\Delta}$ derivable in perturbation theory). For a term containing a product of $u$ creation and $v$ annihilation operators we define a parent momentum, denoted by $P_{u v}^{+}$, which equals half of the sum of momenta labeling all the operators. For each momentum label $k_{i}$, with $i$ running through $u+v$ values, we introduce $x_{i}=k_{i}^{+} / P_{u v}^{+}$and $\kappa_{i}^{\perp}=k_{i}^{\perp}-x_{i} P_{u v}^{\perp}$. The regularization factor is defined as

$$
r_{\Delta}=\prod_{i=1}^{u+v} \exp \frac{-\eta_{i} \kappa_{i}^{\perp 2}}{\Delta^{2}}
$$

where $\eta_{i}=\eta\left(x_{i}\right)$ and $\eta$ is a useful function of its argument. One natural choice is $\eta(x)=1$, for it is simple. Another choice is $\eta(x)=1 / x$, a natural one because it appears in invariant 
masses. We shall assume the function $\eta$ lies between the two choices but otherwise it will be left unspecified. Leaving $\eta$ unspecified will help us identify finite regularization effects.

Consequently, the regularization factor in the second term in Eq. (2.7) is

$$
r_{\Delta}=\exp \frac{-\left(\eta_{1}+\eta_{2}\right) \kappa_{12}^{\perp 2}}{\Delta^{2}}
$$

where $x_{1}=k_{1}^{+} / k_{3}^{+}$and $\kappa_{12}^{\perp}=k_{1}^{\perp}-x_{1} k_{3}^{\perp}$.

The initial Hamiltonian of the similarity renormalization group procedure in $\phi^{3}$ theory in 6 dimensions is given by Eq. (2.7). In order to derive the third order running coupling constant in effective Hamiltonians, we have to calculate $X_{\Delta}$ up to the terms order $g^{3}$. The counterterms are calculated order by order along the evaluation of renormalization group flow for effective Hamiltonians.

\subsection{Similarity Flow of Hamiltonians}

The effective Hamiltonians are written as

$$
H_{\lambda}=F_{\lambda}\left[G_{\lambda}\right]
$$

where

$$
G_{\lambda}=\mathcal{U}_{\lambda} \mathcal{G}_{\lambda} \mathcal{U}_{\lambda}^{\dagger}
$$

The operator $\mathcal{G}_{\lambda}$ is divided into two parts, $\mathcal{G}_{\lambda}=\mathcal{G}_{0}+\mathcal{G}_{I \lambda}$, where $\mathcal{G}_{0}=\mathcal{G}_{\infty}(g=0)$. $\mathcal{G}_{I \lambda}$ satisfies the following differential equation as a consequence of Eqs. (1.3a)-(1.4) (see Ref. [14])

$$
\frac{d}{d \lambda} \mathcal{G}_{I \lambda}=\left[f \mathcal{G}_{I},\left\{\frac{d}{d \lambda}(1-f) \mathcal{G}_{I}\right\}_{\mathcal{G}_{0}}\right] \text {. }
$$

We dropped the subscript $\lambda$ on the right-hand side for clarity. $f$ denotes the similarity form factor introduced by $F_{\lambda}$ and the curly bracket with the subscript $\mathcal{G}_{0}$ denotes a solution for $\mathcal{T}_{\lambda}$ resulting from Eq. (1.4). We will omit the subscript $\mathcal{G}_{0}$ from now on and the Reader should remember that a curly bracket implies an energy denominator for every term in the bracket, i.e. a factor equal to inverse of the eigenvalue of $\mathcal{G}_{0}$ corresponding to momentum labels of all annihilation operators in a term minus eigenvalue of $\mathcal{G}_{0}$ corresponding to all creation operators in the term.

The similarity factor is defined for any operator of the form described above Eq. (2.8) as

$$
f_{\lambda}(u, v)=\exp \left[-\left(\mathcal{M}_{u}^{2}-\mathcal{M}_{v}^{2}\right)^{2} / \lambda^{4}\right] .
$$

The script notation for invariant masses means $\mathcal{M}_{u}^{2}=\left(k_{1}+\ldots+k_{u}\right)^{2}$, where the minus components of the momentum four-vectors are given by $k_{i}^{-}=\left(k_{i}^{\perp}+\mu^{2}\right) / k_{i}^{+}$for $i=1, \ldots, u$.

Let us denote differentiation with respect to $\lambda$ by a prime and expand the effective interaction in powers of the coupling constant $g$ as

$$
\mathcal{G}_{I}=\sum_{n=1}^{\infty} \tau_{n},
$$


where $\tau_{n}$ denotes the sum of all terms order $g^{n}$ in $\mathcal{G}_{\lambda}$. Equation (2.12) implies

$$
\tau_{n}^{\prime}=\sum_{k=1}^{n-1}\left[\tau_{k},\left\{(1-f) \tau_{n-k}\right\}^{\prime}\right],
$$

and the first three terms in the expansion satisfy the following equations

$$
\begin{gathered}
\tau_{1}^{\prime}=0 \\
\tau_{2}^{\prime}=\left[\left\{f^{\prime} \tau_{1}\right\}, f \tau_{1}\right], \\
\tau_{3}^{\prime}=\left[f \tau_{1},\left\{-f^{\prime} \tau_{2}+(1-f) \tau_{2}^{\prime}\right\}\right]+\left[f \tau_{2},\left\{-f^{\prime} \tau_{1}\right\}\right] .
\end{gathered}
$$

Equation (2.16a) implies that $\tau_{1}$ is independent of $\lambda$ and equals the second term in the initial Hamiltonian from Eq. (2.7). In other words, $\tau_{\lambda 1}=\tau_{\infty 1}$. The corresponding effective Hamiltonian interaction term is obtained by multiplying the integrand in Eq. (2.7) by $f_{\lambda}(12,3)=\exp \left\{-\left[\left(k_{1}+k_{2}\right)^{2}-\mu^{2}\right]^{2} / \lambda^{4}\right\}$ and transforming operators $a_{\infty k}$ into $a_{\lambda k}$ using $\mathcal{U}_{\lambda}$ after the evaluation process for $\mathcal{G}_{\lambda}$ is carried out to the desired order.

This last step is unusual in the sense that the operator $\mathcal{U}_{\lambda}$ depends on the regularization (in scalar theory, the dependence is reduced to $\mathcal{U}_{\lambda}$ being a functional of $r_{\Delta}$ ) but, at the same time it is unitary and thus, it transforms finite products of creation and annihilation operators by effectively replacing everywhere $a_{\infty}$ by $a_{\lambda}$ and no other trace of $\mathcal{U}_{\lambda}$ is left in the effective Hamiltonian. If $\mathcal{G}_{\lambda}$ is found to order $g^{n}$, leaving terms $o\left(g^{n+1}\right)$ still undetermined, then $\mathcal{T}_{\lambda}$ is determined up to terms order $g^{n}$ from Eq. (1.4). $\mathcal{T}_{\lambda}$ is antihermitian order by order and determines a unitary $\mathcal{U}_{\lambda}$ to order $g^{n}$, denoted $\mathcal{U}_{\lambda}^{(n)}$. Therefore,

$$
\mathcal{U}_{\lambda}^{(n)} \prod_{i \in u} a_{\infty i}^{\dagger} \prod_{j \in v} a_{\infty j} \mathcal{U}_{\lambda}^{(n) \dagger}=\prod_{i \in u} a_{\lambda i}^{\dagger} \prod_{j \in v} a_{\lambda j}+o\left(g^{n+1}\right)
$$

and the regularization dependence of $\mathcal{U}_{\lambda}$ does not show up in $H_{\lambda}$ to order $g^{n}$ once $\mathcal{G}_{\lambda}$ is renormalized to order $g^{n}$. In successive orders, by construction, the counterterms in $\mathcal{G}_{\infty}$ preserve unitarity of $\mathcal{U}_{\lambda}$. A perturbative proof of renormalizability for effective Hamiltonians would require demonstration that there exists a set of counterterms that remove regularization dependence from finite momentum parts of $\mathcal{G}_{\lambda}$ to all orders of perturbation theory when $\Delta \rightarrow \infty$ (c.f. [3]) and that the resulting theory predicts covariant results. The present article does not demonstrate such set exists in the case of $\phi^{3}$ theory. Also, our calculation is limited to terms order $g, g^{2}$ and $g^{3}$. To verify that an effective Hamiltonian containing a running coupling constant order $g^{3}$ may produce covariant results for scattering processes, the present calculation must be extended to 4 th order.

The transformation connecting two effective Hamiltonians with different finite widths $\lambda_{1}$ and $\lambda_{2}$ is given by $\mathcal{U}_{\lambda_{1}} \mathcal{U}_{\lambda_{2}}^{\dagger}$. One can easily see that the latter is free from dependence on $r_{\Delta}$ once $\mathcal{G}_{\lambda}$ is made independent of $r_{\Delta}$. It is sufficient to observe that for an infinitesimal difference between $\lambda_{1}$ and $\lambda_{2}$ the effective transformation $\mathcal{U}_{\lambda_{1}} \mathcal{U}_{\lambda_{2}}^{\dagger}$ is given by the similarity generator that is expressed in terms of the $r_{\Delta}$-independent $\mathcal{G}_{\lambda}$. Integrating the infinitesimal changes one obtains the same conclusion for finite changes of the width. 
Evaluation of $\tau_{2}$ and $\tau_{3}$ involves calculation of two counterterms. Both $\tau_{2}$ and $\tau_{3}$ are more complicated in structure than $\tau_{1}$ and their evaluation requires new notation. Namely,

$$
\begin{gathered}
\tau_{1}=\alpha_{21}+\alpha_{12}, \\
\tau_{2}=\beta_{11}+\beta_{31}+\beta_{13}+\beta_{22}, \\
\tau_{3}=\gamma_{21}+\gamma_{12}+\gamma_{41}+\gamma_{14}+\gamma_{32}+\gamma_{23} .
\end{gathered}
$$

Each term contains products of creation and annihilation operators with fixed numbers of the operators in a product. The first subscript indicates the number of creation operators, $a_{\infty}^{\dagger}$, and the second subscript denotes the number of annihilation operators, $a_{\infty}$. For all the terms, $\pi_{u v}=\pi_{v u}^{\dagger}$.

Equations (2.16a-b) imply for second order terms the following relations [14],

$$
\begin{aligned}
\beta_{31}^{\prime} & =2 f_{2}\left[\alpha_{21} \alpha_{21}\right]_{31}, \\
\beta_{13}^{\prime} & =2 f_{2}\left[\alpha_{12} \alpha_{12}\right]_{13}, \\
\beta_{11}^{\prime} & =2 f_{2}\left[\alpha_{12} \alpha_{21}\right]_{11}, \\
\beta_{22}^{\prime}= & f_{2}\left[\alpha_{21} \alpha_{12}+4 \alpha_{12} \alpha_{21}\right]_{22} .
\end{aligned}
$$

The brackets mean replacement of products $a_{i} a_{j}^{\dagger}$ by commutators $\left[a_{i}, a_{j}^{\dagger}\right]$. The remaining products of operators contain as many creation and annihilation operators as indicated by the bracket subscripts, in the normal order, according to the same convention as in Eqs. (2.18ac). The factor $f_{2}$ depends on the momenta labeling creation and annihilation operators. In Eqs. (2.19a-d), the brackets with operators involve integrals over three-dimensional momentum labels of all creation and annihilation operators and over loop momenta in loops that result from contractions (in second order here only Eq. (2.19c) for $\beta_{11}^{\prime}$ contains a loop integral). The factor $f_{2}$ is understood to appear under the integrals. Symbolically, its structure appears in Eq. (2.16b) and reads $f_{2}=\left\{f^{\prime}\right\} f-f\left\{f^{\prime}\right\}$. The negative sign results from the commutator in Eq. (2.16b) that guarantees that only connected terms appear in the effective interactions. This is a general property of the similarity procedure.

The factor $f_{2}$ is the only factor depending on $\lambda$ on the right-hand side of Eqs. (2.19a-d). Therefore, solutions are

$$
\begin{gathered}
\beta_{\lambda 31}=2 \mathcal{F}_{2 \lambda}\left[\alpha_{21} \alpha_{21}\right]_{31}, \\
\beta_{\lambda 13}=2 \mathcal{F}_{2 \lambda}\left[\alpha_{12} \alpha_{12}\right]_{13}, \\
\beta_{\lambda 11}=2 \mathcal{F}_{2 \lambda}\left[\alpha_{12} \alpha_{21}\right]_{11}+\beta_{\infty 11},
\end{gathered}
$$




$$
\beta_{\lambda 22}=\mathcal{F}_{2 \lambda}\left[\alpha_{21} \alpha_{12}+4 \alpha_{12} \alpha_{21}\right]_{22},
$$

where $\beta_{\infty 11}$ is a counterterm whose structure is shown in the next Section to be

$$
\beta_{\infty 11}=\int[k] \frac{\delta \mu_{\infty}^{2}}{k^{+}} a_{\infty k}^{\dagger} a_{\infty k}
$$

The only new element of solutions $(2.20 \mathrm{a}-\mathrm{d})$ which requires explanation is the factor $\mathcal{F}_{2 \lambda}=$ $\int_{\infty}^{\lambda} f_{2}$. It is given by the following expression, which is a consequence of Eq. (2.13),

$$
\mathcal{F}_{2 \lambda}(a, b, c)=\frac{P_{b a}^{+} b a+P_{b c}^{+} b c}{b a^{2}+b c^{2}}\left[f_{\lambda}(a, b) f_{\lambda}(b, c)-1\right] .
$$

Arguments $a, b$ and $c$ denote three successive momentum configurations appearing in brackets in Eqs. (2.19a-d) in the order from the left to right, i.e. a denotes the momenta labeling creation operators in the brackets, $c$ denotes the momenta labeling annihilation operators and $b$ denotes the intermediate configuration, which includes the momenta labeling operators contracted in the brackets and momenta labeling creation operators originating from the interaction connecting configuration $b$ with $c$ and momenta labeling annihilation operators originating in the interaction connecting configuration $a$ with $b$.

$P_{u v}^{+}$denotes parent momentum for the whole connected interaction sequence between momentum configurations $u$ and $v$ (in the second order case here the sequence reduces to a single interaction vertex order $g$, but the definition of $P_{u v}^{+}$remains valid in higher order cases later). The symbols $b a$ and $b c$ denote differences of invariant masses, as explained below. We use abbreviations $b a^{2} \equiv(b a)^{2}$ etc.

For any two momentum configurations $u$ and $v$

$$
u v=\mathcal{M}_{u v}^{2}-\mathcal{M}_{v u}^{2}
$$

where

$$
\mathcal{M}_{u v}^{2}=\left[\sum_{i \in u(v)} k_{i}\right]^{2}
$$

and $u(v)$ denotes those momenta from the configuration $u$ that are involved in interactions acting between the configurations $u$ and $v$. Minus components of all momenta are given by $k^{-}=\left(k^{\perp 2}+\mu^{2}\right) / k^{+}$. As an example of this notation, the similarity form factor from Eq. (2.13) reads

$$
f_{\lambda}(u, v)=\exp \frac{-v u^{2}}{\lambda^{4}}
$$

Equation (2.16c) for third order interactions implies the following result for terms relevant to the running coupling constant evaluation,

$$
\begin{aligned}
& \gamma_{21}^{\prime}=f_{3}\left[8\left[\alpha_{12} \alpha_{21} \alpha_{21}\right]_{21}+4\left[\left[\alpha_{12} \alpha_{21}\right] \alpha_{21}\right]_{21}+2\left[\alpha_{21}\left[\alpha_{12} \alpha_{21}\right]\right]_{21}\right]+ \\
& -2\left\{f^{\prime}\right\}\left[\beta_{\infty 11} \alpha_{21}\right]_{21}+\left\{f^{\prime}\right\}\left[\alpha_{21} \beta_{\infty 11}\right]_{21} .
\end{aligned}
$$


The factor $f_{3}$ has the structure $f_{3}=\left[f\left\{(1-f) \mathcal{F}_{2}\right\}^{\prime}+\left\{f^{\prime}\right\} f \mathcal{F}_{2}\right]-\left[\left\{(1-f) \mathcal{F}_{2}\right\}^{\prime} f+f \mathcal{F}_{2}\left\{f^{\prime}\right\}\right]$. Integration of both sides of Eq. (2.24) gives

$$
\begin{aligned}
& \gamma_{\lambda 21}=\mathcal{F}_{3 \lambda}\left[8\left[\alpha_{12} \alpha_{21} \alpha_{21}\right]_{21}+4\left[\left[\alpha_{12} \alpha_{21}\right] \alpha_{21}\right]_{21}+2\left[\alpha_{21}\left[\alpha_{12} \alpha_{21}\right]\right]_{21}\right]+ \\
& +2\left\{1-f_{\lambda}\right\}\left[\beta_{\infty 11} \alpha_{21}\right]_{21}-\left\{1-f_{\lambda}\right\}\left[\alpha_{21} \beta_{\infty 11}\right]_{21}+\gamma_{\infty 21}
\end{aligned}
$$

where $\gamma_{\infty 21}$ denotes the third order counterterm, to be calculated in the next Section. A new element of Eq. (2.25) is the factor $\mathcal{F}_{3 \lambda}=\int_{\infty}^{\lambda} f_{3}$. It appears in front of operator brackets that involve four successive momentum configurations denoted from the left to right by $a, b, c$ and $d$. The brackets contain one loop integral. The configuration $a$ has two momenta, $k_{1}$ and $k_{2}$, while the configuration $d$ only one, $k_{3}[c . f$. Eq. (2.7)]. We have

$$
\mathcal{F}_{3 \lambda}(a, b, c, d)=F_{3}(a, b, c, d)+F_{3}(d, c, b, a)
$$

where

$$
\begin{aligned}
& F_{3}(a, b, c, d)=\frac{P_{c b}^{+} c b+P_{c d}^{+} c d}{c b^{2}+c d^{2}}\left[\left(P_{b d}^{+} b d+P_{b a}^{+} b a\right)\left[\frac{f_{a b} f_{b c} f_{c d} f_{b d}-1}{a b^{2}+b c^{2}+c d^{2}+b d^{2}}-\frac{f_{a b} f_{b d}-1}{a b^{2}+b d^{2}}\right]+\right. \\
& \left.+P_{b d}^{+} \frac{b c^{2}+c d^{2}}{d b}\left[\frac{f_{a b} f_{b c} f_{c d}-1}{a b^{2}+b c^{2}+c d^{2}}-\frac{f_{a b} f_{b c} f_{c d} f_{b d}-1}{a b^{2}+b c^{2}+c d^{2}+b d^{2}}\right]\right]
\end{aligned}
$$

and $f_{a b}$ is an abbreviated notation for $f_{\lambda}(a, b)=\exp \left[-a b^{2} / \lambda^{4}\right]$; c.f. Eq. (2.23c) above.

\section{COUNTERTERMS THROUGH ORDER $g^{3}$}

The two counterterms, $\beta_{\infty 11}$ from Eq. (2.20c) and $\gamma_{\infty 21}$ from Eq. (2.25), are calculated using the equations they appear in. The counterterms are determined by the condition that those equations are independent of $r_{\Delta}$ when $\Delta \rightarrow \infty$ for arbitrary finite values of $\lambda$ and particle momenta. We first describe calculation of $\beta_{\infty 11}$ and then $\gamma_{\infty 21}$.

\subsection{Mass counterterm}

Equation (2.20c) implies

$$
\beta_{\lambda 11}=\int[k] \frac{\delta \mu_{\lambda}^{2}}{k^{+}} a_{\infty k}^{\dagger} a_{\infty k}
$$

where 


$$
\delta \mu_{\lambda}^{2}=\delta \mu_{\infty}^{2}+\left(\frac{g}{2}\right)^{2} \frac{1}{2(2 \pi)^{5}} \int_{0}^{1} \frac{d x}{x(1-x)} \int d^{4} \kappa^{\perp} \frac{2}{\mathcal{M}^{2}-\mu^{2}}\left[f_{\lambda}^{2}\left(\mathcal{M}^{2}, \mu^{2}\right)-1\right] r_{\Delta \beta}
$$

The script $\mathcal{M}$ denotes invariant mass, $\mathcal{M}^{2}=\left(\kappa^{\perp 2}+\mu^{2}\right) / x(1-x)$, and the regularization factor comes out to be

$$
r_{\Delta \beta}=\exp \left\{-2[\eta(x)+\eta(1-x)] \kappa^{\perp 2} / \Delta^{2}\right\} .
$$

$\delta \mu_{\infty}^{2}$ in Eq. (3.2a) is the counterterm contribution. The counterterm is of the form given in Eq. (2.21) since the integral in the second term in Eq. (3.2a) is independent of the momentum $k$ and depends on regularization. The result of integration depends on the cutoff parameter $\Delta$ and function $\eta$. A finite, regularization dependent part of the result remains undetermined. Therefore, we have to adjust its value to data.

Without loss of generality, we assume that some gedanken data requires the mass squared parameter in effective Hamiltonian with $\lambda=\lambda_{0}$ to be equal $\mu^{2}+\delta \mu_{0}^{2}$. Hence,

$$
\delta \mu_{\infty}^{2}=\delta \mu_{0}^{2}-\left(\frac{g}{2}\right)^{2} \frac{1}{2(2 \pi)^{5}} \int_{0}^{1} \frac{d x}{x(1-x)} \int d^{4} \kappa^{\perp} \frac{2}{\mathcal{M}^{2}-\mu^{2}}\left[f_{\lambda_{0}}^{2}\left(\mathcal{M}^{2}, \mu^{2}\right)-1\right] r_{\Delta \beta}
$$

Integration gives two diverging terms, one proportional to $\Delta^{2}$ and another one proportional to $\log \Delta$. The remaining finite part depends on our choice of the function $\eta$. Evaluating the integral for $\eta(x)=1 / x$ one obtains

$$
\delta \mu_{\infty}^{2}=g^{2} \frac{1}{(4 \pi)^{3}}\left[\frac{1}{24} \Delta^{2}-\mu^{2} \frac{5}{6} \log \frac{\Delta}{\mu}+\mu_{\eta}^{2}\right],
$$

where $\mu_{\eta}$ has a finite limit when $\Delta \rightarrow \infty$. The logarithmically divergent part is independent of the function $\eta$ and agrees with results for the Lagrangian mass squared counterterm obtained using Feynman diagrams and dimensional regularization [27] 28] in the following sense: when one changes $\Delta$ to $\Delta^{\prime}$ the logarithmic part of the counterterm changes with $\Delta$ as the mass squared changes as a function of the renormalization scale in Eq. (7.1.22) in [28].

The resulting mass squared term in the effective Hamiltonian can be written in the limit $\Delta \rightarrow \infty$ as

$$
\begin{aligned}
& \mu_{\lambda}^{2}=\mu^{2}+\delta \mu_{\lambda}^{2}= \\
& =\mu^{2}+\delta \mu_{0}^{2}+\left(\frac{g}{2}\right)^{2} \frac{1}{2(2 \pi)^{5}} \int_{0}^{1} \frac{d x}{x(1-x)} \int d^{4} \kappa^{\perp} \frac{2}{\mathcal{M}^{2}-\mu^{2}}\left[f_{\lambda}^{2}\left(\mathcal{M}^{2}, \mu^{2}\right)-f_{\lambda_{0}}^{2}\left(\mathcal{M}^{2}, \mu^{2}\right)\right] .
\end{aligned}
$$

The above result is particularly simple for $\mu=0$ and in that case it reads $\left(\delta \mu_{0}^{2}\right.$ is proportional to $\left.g^{2}\right)$ 


$$
\mu_{\lambda}^{2}=\delta \mu_{0}^{2}+g^{2} \frac{1}{(4 \pi)^{3}} \frac{1}{24} \sqrt{\frac{\pi}{2}}\left(\lambda^{2}-\lambda_{0}^{2}\right)
$$

Logarithmic dependence on $\lambda$ arises for $\mu>0$. The value of $\delta \mu_{0}^{2}$ could be found, for example, by solving a single physical meson eigenvalue problem, expressing the physical meson mass in terms of $\delta \mu_{0}^{2}$ and adjusting the latter to obtain the gedanken experimental mass value for mesons.

\subsection{Coupling constant counterterm}

The coupling constant counterterm $\gamma_{\infty 21}$ is evaluated from Eq. (2.25). The mass squared counterterms cancel quadratic and part of logarithmic divergences so that the vertex counterterm is needed to remove only logarithmically divergent and finite regularization effects.

The interaction term $\gamma_{\lambda 21}$ has the form

$$
\gamma_{\lambda 21}=\int\left[k_{1} k_{2} k_{3}\right] 2(2 \pi)^{5} \delta^{5}\left(k_{1}+k_{2}-k_{3}\right)\left[\gamma_{\lambda}\left(k_{1}, k_{2}, k_{3}\right)+\gamma_{\infty}\left(k_{1}, k_{2}, k_{3}\right)\right] a_{\infty k_{1}}^{\dagger} a_{\infty k_{2}}^{\dagger} a_{\infty k_{3}} r_{\Delta} .
$$

The counterterm function $\gamma_{\infty}\left(k_{1}, k_{2}, k_{3}\right)$ originates from $\gamma_{\infty 21}$ in Eq. (2.25). Since the similarity renormalization group procedure preserves canonical symmetries of light-front Hamiltonians, the functions $\gamma_{\lambda}\left(k_{1}, k_{2}, k_{3}\right)$ and $\gamma_{\infty}\left(k_{1}, k_{2}, k_{3}\right)$ depend only on variables $x_{1}$ and $\kappa_{12}^{\perp}$, introduced in Eq. (2.9).

The entire regularization dependence of $\gamma_{\lambda}\left(k_{1}, k_{2}, k_{3}\right) \equiv \gamma_{\lambda}\left(x_{1}, \kappa_{12}^{\perp}\right)$ in the limit $\Delta \rightarrow \infty$ is contained in

$$
\begin{aligned}
& \left.\left.\gamma_{\lambda}\left(x_{1}, \kappa_{12}^{\perp}\right)\right|_{r_{\Delta}} \equiv \gamma_{\lambda}\left(x_{1}, 0^{\perp}\right)\right|_{r_{\Delta}}=\left(\frac{g}{2}\right)^{3} \frac{\pi^{2}}{2(2 \pi)^{5}} \times \\
& \times\left[\frac{1}{2}\left[\int_{x_{1}}^{1} \frac{d x}{x(1-x)\left(x-x_{1}\right)} \int_{0}^{\infty} \kappa^{2} d \kappa^{2} 8 \frac{x-x_{1}}{x x_{2} \mathcal{M}^{4}} \exp \left(\frac{-c_{\eta} \kappa^{2}}{\Delta^{2}}\right)+\left(x_{1} \leftrightarrow x_{2}\right)\right]+\right. \\
& \left.+\int_{0}^{1} \frac{d x}{x(1-x)} \int_{0}^{\infty} \kappa^{2} d \kappa^{2} \frac{-3}{\mathcal{M}^{4}} \exp \left(\frac{-d_{\eta} \kappa^{2}}{\Delta^{2}}\right)\right]
\end{aligned}
$$

where

$$
c_{\eta}=\eta(x)+\eta(1-x)+\left\{\eta\left(x_{1} / x\right)+\eta\left[\left(x-x_{1}\right) / x\right]\right\}\left(x_{1} / x\right)^{2}+\eta\left[\left(x-x_{1}\right) / x_{2}\right]+\eta\left[(1-x) / x_{2}\right]
$$

and

$$
d_{\eta}=2[\eta(x)+\eta(1-x)]
$$


The first term in Eq. (3.7a), symmetrized in $x_{1}$ and $x_{2}$, originates from the first 8 terms in the long bracket in Eq. (2.25). The second term originates from the next $4+2$ terms in the long bracket and $2+1$ mass counterterm terms in Eq. (2.25). The regularization effects are independent of $\kappa_{12}^{\perp}$. The apparent singularity at $\kappa^{2} \rightarrow 0$ for $\mu=0$ is irrelevant to the regularization dependence and appears here only because we do not display similarity factors that remove the singularity. The full expression for $\gamma_{\lambda}\left(x_{1}, \kappa_{12}^{\perp}\right)$ is given in Appendix.

Dropping all parts that are independent of regularization, Eq. (3.7a) gives

$$
\begin{aligned}
& \left.\gamma_{\lambda}\left(x_{1}, 0^{\perp}\right)\right|_{r_{\Delta}}=\left(\frac{g}{2}\right)^{3} \frac{1}{(4 \pi)^{3}} \times \\
& {\left[3 \log \frac{\Delta}{\mu}-4\left[\int_{x_{1}}^{1} d x \frac{1-x}{x_{2}} \log c_{\eta}+\left(x_{1} \leftrightarrow x_{2}\right)\right]+3 \int_{0}^{1} d x x(1-x) \log d_{\eta}\right] .}
\end{aligned}
$$

The bare boson mass, $\mu$, can be replaced by a finite constant of the same dimension in the case of massless bosons.

Equation (3.8) says that the diverging regularization dependence of the interaction vertex, i.e. the term proportional to $\log \Delta$, is independent of the particle momenta and one can remove the divergence by merely changing the initial coupling constant $g$ in Eq. (2.7). Thus, no diverging $x$-dependent counterterms are required - different situation than in [29]. However, it is visible that the vertex contains a finite regularization dependent part that is a function of $x_{1}$. The function depends on our choice for $\eta$. One could completely subtract the $\eta$-dependence for arbitrary choices of $\eta$ by defining a counterterm that contains a negative of the $\eta$-dependent part of the right-hand side of Eq. (3.8). For example, if $\eta=1$ one has $c_{\eta}=4+2\left(x_{1} / x\right)^{2}$ and $d_{\eta}=4$. Integration produces a smooth and slowly varying function of $x_{1}$, which needs to be subtracted.

Since the whole regularization effect is independent of $\lambda$ and $\kappa_{12}^{\perp}$, it can be completely removed from $\gamma_{\lambda}\left(x_{1}, \kappa_{12}^{\perp}\right)$ by subtracting $\gamma_{\lambda_{0}}\left(x_{1}, 0^{\perp}\right)$, where $\lambda_{0}$ is chosen arbitrarily. However, one has to add back the finite regularization independent part of $\gamma_{\lambda_{0}}\left(x_{1}, 0^{\perp}\right)$, which will be denoted below by $\gamma_{0}\left(x_{1}\right)$. The function $\gamma_{0}\left(x_{1}\right)$ is necessary to recover Poincaré symmetry of observables. Regularization spoils Poincaré symmetry. The symmetry may be restored once counterterms remove regularization effects, but one is not allowed to change terms independent of regularization. Therefore, the function $\gamma_{0}\left(x_{1}\right)$ must be reinserted. This function is not altered when $\lambda$ changes and could be considered marginal in analogy with usual renormalization group analysis.

Although one can isolate finite $\eta$-dependent functions of $x_{1}$ in the effective vertex, for the particular choice of the regularization factors we adopt, the ultimate adjustment of the function $\gamma_{0}\left(x_{1}\right)$ has to be delayed until 4 th order calculations are completed. For there exists in $\phi^{3}$ theory no 3rd order scattering amplitude one could use to find out what function $\gamma_{0}\left(x_{1}\right)$ renders Poincaré symmetry of scattering observables with our choice of $r_{\Delta}$ in Eq. (2.7). It will be interesting to see if the explicit dependence on $\eta$ isolates the same function as required by the symmetry. However, it should be pointed out that the function does not influence the way the 3rd order running coupling constant in effective Hamiltonians depends on $\lambda$. 
The counterterm function $\gamma_{\infty}\left(k_{1}, k_{2}, k_{3}\right) \equiv \gamma_{\infty}\left(x_{1}\right)$, which removes the regularization dependence from the effective vertex reads

$$
\gamma_{\infty}\left(x_{1}\right)=-\gamma_{\lambda_{0}}\left(x_{1}, 0^{\perp}\right)+\gamma_{0}\left(x_{1}\right)
$$

It can be used to define a new regularization dependent coupling constant $g_{\Delta}$ in the initial Hamiltonian in Eq. (2.7). We select a convenient value of $x_{1}=x_{0}$ and obtain

$$
\frac{g_{\Delta}}{2}=\frac{g}{2}+\gamma_{\infty}\left(x_{0}\right)=\frac{g}{2}-\gamma_{\lambda_{0}}\left(x_{0}, 0^{\perp}\right)+\gamma_{0},
$$

where $\gamma_{0} \equiv \gamma_{0}\left(x_{0}\right)$. Using Eq. (3.8), we see that the initial coupling $g$ is replaced by the new $\Delta$-dependent quantity

$$
g_{\Delta}=g\left[1-g^{2} \frac{3}{4(4 \pi)^{3}} \log \frac{\Delta}{m_{0}}\right]+o\left(g^{5}\right) .
$$

with certain constant $m_{0}$. Thus, the theory exhibits asymptotic freedom in 3rd order terms. Our result agrees with literature, say Eq. (7.1.26) from [28], in the sense that when we change $\Delta$, the change required in the coupling constant in the initial Hamiltonian for obtaining $\Delta$ independent effective Hamiltonians matches the change implied by Feynman diagrams and dimensional regularization. Comparison with Feynman calculus will be farther discussed below.

Having derived the structure of counterterms we can proceed to evaluation of the finite similarity flow of effective Hamiltonians towards small widths $\lambda$.

\section{RUNNING COUPLING CONSTANT}

Our procedure for evaluating the running coupling constant in Hamiltonians follows theory from [3] and [4] using [14]. The procedure has been outlined in a matrix model example in [15]. Here, we use particle creation and annihilation operators in a boost invariant way, instead of matrix elements.

The initial Hamiltonian interaction vertex depends on regularization through the factor $r_{\Delta}$ and corresponding counterterm. The bare coupling constant $g$ is replaced by the coupling constant $g_{\Delta}$ according to Eqs. (3.9a-c), i.e.

$$
g_{\Delta}=g-2\left[\gamma_{\lambda_{0}}\left(x_{0}, 0^{\perp}\right)-\gamma_{0}\right] .
$$

The factor 2 is needed because $g / 2$ appears in the Hamiltonian. Both $\gamma_{\lambda_{0}}\left(x_{0}\right)$ and $\gamma_{0}$ are proportional to $g^{3}$. Inverting the series (4.1) one can express $g$ in terms of $g_{\Delta}$.

Evaluation of $\gamma_{\lambda 21}$ in Eq. (3.6) leads now to a finite expression, which has a limit when $\Delta \rightarrow \infty$. In that limit, the effective Hamiltonian interaction term takes the form

$$
H_{\lambda 21}=\int\left[k_{1} k_{2} k_{3}\right] 2(2 \pi)^{5} \delta^{5}\left(k_{1}+k_{2}-k_{3}\right) f_{\lambda}\left[\left(k_{1}+k_{2}\right)^{2}, k_{3}^{2}\right] V_{\lambda}\left(x_{1}, \kappa_{12}^{\perp}\right) a_{\lambda k_{1}}^{\dagger} a_{\lambda k_{2}}^{\dagger} a_{\lambda k_{3}}
$$

where

$$
\left.V_{\lambda}\left(x_{1}, \kappa_{12}^{\perp}\right)=\frac{g_{\Delta}}{2}+\gamma_{\lambda}\left(x_{1}, \kappa_{12}^{\perp}\right)+\gamma_{\lambda_{0}}\left(x_{0}, 0^{\perp}\right)-\gamma_{\lambda_{0}}\left(x_{1}, 0^{\perp}\right)\right]+\gamma_{0}\left(x_{1}\right)-\gamma_{0} \quad,
$$


is the effective vertex function and $f_{\lambda}$ is the similarity vertex form factor. $\gamma_{\lambda}\left(x_{1}, \kappa_{12}^{\perp}\right)$ and the remaining terms in $V_{\lambda}\left(x_{1}, \kappa_{12}^{\perp}\right)$ are proportional to $g^{3}$. Our calculation is done to this order of accuracy only, so that $g^{3} \equiv g_{\Delta}^{3}$ and Eq. (4.2b) is considered to be an expansion in powers of $g_{\Delta}$.

We define the running coupling constant as the value of $2 V_{\lambda}\left(x_{1}, \kappa_{12}^{\perp}\right)$ at a chosen configuration of momentum variables, denoted by $\left(x_{10}, \kappa_{120}^{\perp}\right)$ and specified later. In other words, $g_{\lambda}=2 V_{\lambda}\left(x_{10}, \kappa_{120}^{\perp}\right)$. This is a natural definition, analogous to the standard Thomson limit in the case of electron charge in QED. We have

$$
g_{\lambda}=g_{\Delta}+2\left[\gamma_{\lambda}\left(x_{10}, \kappa_{120}^{\perp}\right)+\gamma_{\lambda_{0}}\left(x_{0}, 0^{\perp}\right)-\gamma_{\lambda_{0}}\left(x_{10}, 0^{\perp}\right)+\gamma_{0}\left(x_{10}\right)-\gamma_{0}\right] .
$$

It is natural to use $x_{0}=x_{10}$. Then,

$$
g_{\lambda}=g_{\Delta}+2 \gamma_{\lambda}\left(x_{10}, \kappa_{120}^{\perp}\right) .
$$

This equation demonstrates that the effective coupling constant $g_{\lambda}$ depends on the value of the finite function $\gamma_{0}\left(x_{1}\right)$ in the counterterm at one point, the same as the one used to define $g_{\Delta}$.

Suppose that for the chosen value of $\lambda=\lambda_{0}$, the running coupling constant should have the value $g_{\lambda_{0}}=g_{0}$, determined from comparison with experiment. Then,

$$
g_{0}=g_{\Delta}+2 \gamma_{\lambda_{0}}\left(x_{10}, \kappa_{120}^{\perp}\right)+o\left(g_{\Delta}^{5}\right),
$$

where $g$ in terms order $g^{3}$ is replaced by $g_{\Delta}$. Inverting this series expansion we obtain

$$
g_{\Delta}=g_{0}-2 \gamma_{\lambda_{0}}\left(x_{10}, \kappa_{120}^{\perp}\right)+o\left(g_{0}^{5}\right),
$$

where in terms order $g_{\Delta}^{3}$ we have $g_{\Delta}$ replaced by $g_{0}$. Relation (4.5) can be inserted into Eq. (4.3b) to yield

$$
g_{\lambda}=g_{0}+2\left[\gamma_{\lambda}\left(x_{10}, \kappa_{120}^{\perp}\right)-\gamma_{\lambda_{0}}\left(x_{10}, \kappa_{120}^{\perp}\right)\right]+o\left(g_{0}^{5}\right)
$$

This relation is free from dependence on the finite function $\gamma_{0}\left(x_{1}\right)$ in the counterterm.

The vertex function in the effective interaction in Eqs. (4.2a-b) is equal

$V_{\lambda}\left(x_{1}, \kappa_{12}^{\perp}\right)=\frac{g_{0}}{2}+\gamma_{\lambda}\left(x_{1}, \kappa_{12}^{\perp}\right)-\gamma_{\lambda_{0}}\left(x_{10}, \kappa_{120}^{\perp}\right)+\gamma_{\lambda_{0}}\left(x_{10}, 0^{\perp}\right)-\gamma_{\lambda_{0}}\left(x_{1}, 0^{\perp}\right)+\gamma_{0}\left(x_{1}\right)-\gamma_{0}+o\left(g_{0}^{5}\right)$,

where in terms order $g^{3}$ the initial $g$ is replaced by $g_{0}$. The difference between $g$ and $g_{0}$ is of order $g_{0}^{3}$ and it is included in terms $o\left(g_{0}^{5}\right)$. Equation (4.7a) gives us the effective vertex function for width $\lambda$ expanded in powers of the effective coupling constant $g_{0}$ corresponding to width $\lambda_{0}$. Written as a power series in $g_{\lambda}$, the effective vertex function reads

$V_{\lambda}\left(x_{1}, \kappa_{12}^{\perp}\right)=\frac{g_{\lambda}}{2}+\gamma_{\lambda}\left(x_{1}, \kappa_{12}^{\perp}\right)-\gamma_{\lambda}\left(x_{10}, \kappa_{120}^{\perp}\right)+\gamma_{\lambda_{0}}\left(x_{10}, 0^{\perp}\right)-\gamma_{\lambda_{0}}\left(x_{1}, 0^{\perp}\right)+\gamma_{0}\left(x_{1}\right)-\gamma_{0}+o\left(g_{\lambda}^{5}\right)$,

where in terms order $g^{3}$ one replaces $g$ by $g_{\lambda}$. Clearly, Eq. (4.7b) reproduces the relation $g_{\lambda}=2 V_{\lambda}\left(x_{10}, \kappa_{120}^{\perp}\right)$. 
It remains to calculate the dependence of $g_{\lambda}$ on $\lambda$, which requires a choice of the momentum configuration $\left(x_{10}, \kappa_{120}^{\perp}\right)$. The particular choice we will adopt is suitable for massless bare bosons, i.e. for $\mu=0$. Our choice would require a change for $\mu>0$, to avoid vanishing of similarity factors when $\mu^{2} / x_{10}$ tends to infinity. But the change is not significant since $\mu>0$ introduces no alteration in our procedure apart from the change of momentum configuration, and it also does not interfere with boost-invariance. Therefore, we will limit details of our presentation to the simplest massless case, using a momentum configuration that is most convenient when $\mu=0$. We also continue to keep $x_{0}$ in Eq. (3.9b) equal to $x_{10}$. For $\mu>0$ one has to make $x_{0}$ greater than 0 , too. Other choices of the momentum configurations than we adopt here are equally possible in the case $\mu=0$. The one we choose is particularly suitable for executing integrals over the loop momenta and extracting the running coupling dependence on the width $\lambda$ analytically. Expressions for $\gamma_{\lambda}\left(x_{1}, \kappa_{12}^{\perp}\right)$ for $\mu \geq 0$ are given in Appendix.

In the massless case, the most convenient configuration is $x_{10}=0$ and $\kappa_{120}^{\perp}=0^{\perp}$. For massive particles one considers $1>x_{10}>0$ but there is no compelling reason to consider $\kappa_{120}^{\perp} \neq 0$ in $\phi^{3}$ theory even for massive particles. Note that the parent momentum $k_{3}$ in Eq. (4.2) is arbitrary and not limited by our choice of the momentum configuration $\left(x_{10}, \kappa_{120}^{\perp}\right)$. This feature is not readily available when one considers only specific matrix elements of effective Hamiltonians or when explicit boost invariance is not preserved by the renormalization group procedure.

Eq. (4.6) gives

$$
\begin{aligned}
& g_{\lambda}=g_{0}+ \\
& +g_{0}^{3} \frac{1}{24} \frac{1}{(4 \pi)^{3}} \int_{0}^{\infty} \frac{d z}{z}\left[2\left(f_{\lambda}-f_{\lambda}^{3}\right)-2\left(f_{0}-f_{0}^{3}\right)+20\left(f_{\lambda}^{3}-f_{\lambda}^{2}\right)-20\left(f_{0}^{3}-f_{0}^{2}\right)+9\left(f_{0}^{2}-f_{\lambda}^{2}\right)\right],
\end{aligned}
$$

where $f_{\lambda}=\exp -z^{2} / \lambda^{4}$ and $f_{0}=\exp -z^{2} / \lambda_{0}^{4}$. A straightforward integration gives

$$
g_{\lambda}=g_{0}-g_{0}^{3} \frac{3}{4(4 \pi)^{3}} \log \frac{\lambda}{\lambda_{0}},
$$

which exhibits asymptotic freedom. Differentiating with respect to $\lambda$ and keeping terms up to order $g_{\lambda}^{3}$ one obtains

$$
\frac{d}{d \lambda} g_{\lambda}=-g_{\lambda}^{3} \frac{3}{256 \pi^{3}} \frac{1}{\lambda}
$$

This equation demonstrates the same $\beta$ function for coupling constants in effective Hamiltonians as obtained in Lagrangian approaches using Feynman diagrams and dimensional regularization, when one identifies the renormalization scale with the Hamiltonian width $\lambda$. This is encouraging but one needs to remember that for comparison of perturbative scattering amplitudes in Hamiltonian and Lagrangian approaches it is necessary to make additional calculations and at least of fourth order in $g$. Beyond model matrix studies such as in [15], 4th order similarity calculations have so far been carried out only in simplified Yukawa model by Masłowski and Więckowski [30].

Integrating Eq. (4.10) one obtains $\left(\alpha=g^{2} / 4 \pi\right)$ 


$$
\alpha_{\lambda}=\frac{\alpha_{0}}{1+\alpha_{0}\left(3 / 32 \pi^{2}\right) \log \lambda / \lambda_{0}}
$$

which shows our result for a boost invariant running coupling constant in effective Hamiltonians. Our procedure explains how the running coupling constant can be included in quantum mechanics of effective particles, which is given by the Schrödinger equation with the corresponding Hamiltonian $H_{\lambda}$.

\section{CONCLUSION}

A remarkable result of the whole procedure in the case of asymptotically free scalar dynamics is an extremely simple structure of diverging counterterms and a complete control over involved effective interactions, in perturbation theory. Our operator calculus preserves cluster properties and allows for evaluation of effective Hamiltonians without limitation to any particular set of matrix elements. In other words, we can easily derive integral expressions for matrix elements of effective Hamiltonians in the whole Fock space spanned by basis states of effective particles. The renormalization group equations are integrated analytically using gaussian similarity form factors. Also, the unitary nature of the similarity transformation for effective particle creation and annihilation operators removes wave function renormalization constant from the procedure. However, the regularization factor introduced in the initial Hamiltonian requires an additional finite counterterm that contains a boost invariant function of longitudinal momentum fractions.

The renormalization group flow for Hamiltonians differs from standard procedures applied to S-matrix. The key difference from standard procedures is that the running coupling constant is derived as a coefficient in front of a certain term in an effective renormalized light-front Hamiltonian instead of a Lagrangian term or in a scattering amplitude. The advantage of knowing an effective Hamiltonian is that not only one can attempt to describe scattering processes but also bound states using the corresponding eigenvalue equation.

Note that the light-front form of relativistic quantum field dynamics re-defines the vacuum problem in a way that is only partly understood. [7] [26] But the present calculation shows that running coupling constant calculations can be consistently carried out in lowest orders of perturbation theory without inclusion of perturbative modifications of the vacuum state. This leads to a new demand for similar perturbative analysis of theories that may include effects usually thought to be associated with ground state properties, the closest being a scalar theory with quartic interaction term and infinitesimally small coupling constant.

Obviously, a more sophisticated treatment is necessary in gauge theories for many reasons but, in particular, because they require an additional cutoff limiting the longitudinal momentum fractions from below. The small- $x$ cutoff appears in the unitary transformation $\mathcal{U}_{\lambda}$ as well as in the transformations $\mathcal{U}_{\lambda_{1}} \mathcal{U}_{\lambda_{2}}^{\dagger}$, the former depending on and the latter being independent of the ultraviolet cutoff $\Delta$. This difference between the small-x cutoff and the ultraviolet cutoff cannot be studied in the case of scalar fields discussed in the present paper. However, the third order boost-invariant similarity factors we derived in the case of $\phi^{3}$ directly apply, for example, in calculation of a triple-gluon vertex in effective QCD.

Finally, we wish to stress the difference between the regularized initial Hamiltonian for bare particles and the small width Hamiltonian for effective particles, which contains similarity form factors $f_{\lambda}$. The form factors dampen interactions changing invariant masses by more 
than $\lambda$ and thus can tame the spread of eigenstate wave functions for low lying eigenvalues into regions of high relative momenta of constituents. This feature may lead to exponential convergence of the eigenstate expansion in the effective particle basis. Such convergence is not expected in the case of bare particles. The fine structure of effective particles would then unfold in the transformation $\mathcal{U}_{\lambda_{1}} \mathcal{U}_{\lambda_{2}}^{\dagger}$ relating effective degrees of freedom at two different scales, one corresponding to the binding scale and the other to the high momentum transfer probe in question. This picture encourages opinion that the present calculation provides a pattern worth trying in application to realistic theories.

\section{Acknowledgments}

The author thanks Ken Wilson for important discussion concerning $x$-dependent counterterms and the manuscript, and Bob Perry for his comments.

\section{Appendix}

The loop integrals in Eq. (2.25) are given in the order implied by first three terms in the first bracket. The first term contains the integral $I_{1}$, the second term in the bracket together with the first mass counterterm term contain $I_{2}$ and the third term in the bracket together with the second mass counterterm contain $I_{3}$. The right-hand side of Eq. (2.25) is given by Eq. (3.6) where $\gamma_{\lambda}\left(k_{1}, k_{2}, k_{3}\right)=I_{1}+I_{2}+I_{3}$. In all three terms we have

$$
\mathcal{M}_{12}^{2}=\left(\kappa_{12}^{\perp 2}+\mu^{2}\right) /\left(x_{1} x_{2}\right)
$$

where

$$
x_{1}=k_{1}^{+} / k_{3}^{+},
$$

and

$$
\kappa_{12}^{\perp}=k_{1}^{\perp}-x_{1} k_{3}^{\perp}
$$

The first term integral is

$$
I_{1}=\left(\frac{g}{2}\right)^{2} \frac{1}{2(2 \pi)^{5}} \frac{1}{2}\left[\int_{x_{1}}^{1} \frac{d x}{x(1-x)\left(x-x_{1}\right)} \int d^{4} \kappa^{\perp} \frac{1}{k_{3}^{+2}} 8 \mathcal{F}_{3 \lambda}(a, b, c, d) r_{\Delta 1} \quad+\quad(1 \leftrightarrow 2)\right]
$$

where in $\mathcal{F}_{3 \lambda}(a, b, c, d)$ in Eqs. (2.26a-b) one substitutes

$$
\begin{gathered}
a b=-b a=\mu^{2}-\mathcal{M}_{68}^{2}, \\
a c=-c a=\mathcal{M}_{12}^{2}-\mathcal{M}^{2}, \\
a d=-d a=\mathcal{M}_{12}^{2}-\mu^{2},
\end{gathered}
$$




$$
\begin{gathered}
b c=-c b=\mathcal{M}_{16}^{2}-\mu^{2}, \\
b d=-d b=b c / x+c d=b a / x_{2}+a d, \\
c d=-d c=\mathcal{M}^{2}-\mu^{2}
\end{gathered}
$$

with

$$
\begin{gathered}
\mathcal{M}^{2}=\left(\kappa^{\perp 2}+\mu^{2}\right) /[x(1-x)] \\
\mathcal{M}_{16}^{2}=x^{2}\left(\kappa_{16}^{\perp 2}+\mu^{2}\right) /\left[\left(x-x_{1}\right) x_{1}\right], \\
\mathcal{M}_{68}^{2}=x_{2}^{2}\left(\kappa_{68}^{\perp 2}+\mu^{2}\right) /\left[\left(x-x_{1}\right)(1-x)\right], \\
r_{\Delta 1}=\exp \left\{-\left[[\eta(x)+\eta(1-x)] \kappa^{\perp 2}+\left[\eta\left[\left(x-x_{1}\right) / x\right]+\eta\left(x_{1} / x\right)\right] \kappa_{16}^{\perp 2}+\right.\right. \\
\left.\left.+\left[\eta\left[\left(x-x_{1}\right) / x_{2}\right]+\eta\left[(1-x) / x_{2}\right]\right] \kappa_{68}^{\perp 2}\right] / \Delta^{2}\right\}
\end{gathered}
$$

and

$$
\begin{gathered}
\kappa_{16}^{\perp}=\kappa_{12}^{\perp}-x_{1} \kappa^{\perp} / x \\
\kappa_{68}^{\perp}=\kappa^{\perp}-(1-x) \kappa_{12}^{\perp} / x_{2} \\
P_{a b}^{+}=P_{b a}^{+}=x_{2} k_{3}^{+}, \\
P_{b c}^{+}=P_{c b}^{+}=x k_{3}^{+}, \\
P_{b d}^{+}=P_{d b}^{+}=P_{c a}^{+}=P_{a c}^{+}=P_{c d}^{+}=P_{d c}^{+}=k_{3}^{+}
\end{gathered}
$$

The expression for $I_{2}$ is

$$
\begin{aligned}
& I_{2}=\frac{1}{2}\left[( \frac { g } { 2 } ) ^ { 2 } \frac { 1 } { 2 ( 2 \pi ) ^ { 5 } } \int _ { 0 } ^ { 1 } \frac { d x } { x ( 1 - x ) } \int d ^ { 4 } \kappa ^ { \perp } \left[\frac{1}{k_{2}^{+2}} 4 \mathcal{F}_{3 \lambda}(a, b, c, d)\right.\right. \\
& \left.\left.-2 \frac{f_{c d}-1}{d c} \frac{1}{x_{2}}\left[-\frac{2}{k_{2}^{+}} \mathcal{F}_{2 \lambda_{0}}(a, b, c)\right]\right] r_{\Delta 2}-2 \frac{f_{a d}-1}{d a} \frac{g}{2} \frac{\delta \mu_{0}^{2}}{x_{2}}+(1 \leftrightarrow 2)\right],
\end{aligned}
$$

where in $\mathcal{F}_{2 \lambda_{0}}(a, b, c)$ in Eq. $(2.22)$ and $\mathcal{F}_{3 \lambda}(a, b, c, d)$ in Eqs. (2.26a-b) one substitutes 


$$
\begin{gathered}
a b=-b a=\mu^{2}-\mathcal{M}^{2} \\
a c=-c a=0 \\
a d=-d a=c d \\
b c=-c b=b a \\
b d=-d b=b c / x_{2}+c d \\
c d=-d c=\mathcal{M}_{12}^{2}-\mu^{2}
\end{gathered}
$$

with the same

$$
\mathcal{M}^{2}=\left(\kappa^{\perp 2}+\mu^{2}\right) /[x(1-x)],
$$

and

$$
\begin{gathered}
r_{\Delta 2}=\exp \left\{-2[\eta(x)+\eta(1-x)] \kappa^{\perp 2} / \Delta^{2}\right\}, \\
P_{a b}^{+}=P_{a c}^{+}=P_{b c}^{+}=x_{2} k_{3}^{+}, \\
P_{a d}^{+}=P_{b d}^{+}=P_{c d}^{+}=k_{3}^{+} .
\end{gathered}
$$

The expression for $I_{3}$ is

$$
\begin{aligned}
& I_{3}=\left(\frac{g}{2}\right)^{2} \frac{1}{2(2 \pi)^{5}} \int_{0}^{1} \frac{d x}{x(1-x)} \int d^{4} \kappa^{\perp}\left[\frac{1}{k_{3}^{+2}} 2 \mathcal{F}_{3 \lambda}(a, b, c, d)\right. \\
& \left.+\frac{f_{a d}-1}{d a}\left[-\frac{2}{k_{3}^{+}} \mathcal{F}_{2 \lambda_{0}}(b, c, d)\right]\right] r_{\Delta 3}+\frac{f_{a d}-1}{d a} \frac{g}{2} \delta \mu_{0}^{2}
\end{aligned}
$$

where in $\mathcal{F}_{2 \lambda_{0}}(b, c, d)$ in Eq. $(2.22)$ and $\mathcal{F}_{3 \lambda}(a, b, c, d)$ in Eqs. (2.26a-b) one substitutes

$$
\begin{gathered}
a b=a d=\mathcal{M}_{12}^{2}-\mu^{2}, \\
a c=\mathcal{M}_{12}^{2}-\mathcal{M}^{2}, \\
b c=d c=\mu^{2}-\mathcal{M}^{2}
\end{gathered}
$$




$$
b d=0,
$$

with the same $\mathcal{M}^{2}$ as for $I_{1}$ and $I_{2}$ and

$$
r_{\Delta 3}=r_{\Delta 2}
$$

and all parent momenta equal $k_{3}$.

\section{References}

[1] K. Wilson, Phys. Rev. 140, B445 (1965).

[2] K. G. Wilson, Phys. Rev. D2, 1438 (1970).

[3] St. D. Głazek and K. G. Wilson, Phys. Rev. D48, 5863 (1993).

[4] St. D. Głazek and K. G. Wilson, Phys. Rev. D49, 4214 (1994).

[5] P. A. M. Dirac, Rev. Mod. Phys. 21, 392 (1949).

[6] G. P. Lepage and S. J. Brodsky, Phys. Rev. D22, 2157 (1980).

[7] K. G. Wilson et al., Phys. Rev. D49, 6720 (1994); and references therein.

[8] S. J. Brodsky, H. C. Pauli, S. S. Pinsky, Phys. Rep. 301, 299 (1998).

[9] M. Burkardt, Adv. Nucl. Phys. 23, 1 (1996), hep-th/9505259; A. Harindranath, in LightFront Quantization and Non-Perturbative QCD, Proceedings, edited by J. P. Vary and F. Wölz, IITAP (Iowa State University Press, Ames, 1997), hep-ph/9612244; R. J. Perry, in Confinement, Duality and Nonperturbative Aspects of $Q C D$, Proceedings, edited by P. van Baal (Plenum Press, 1998), in series NATO ASI Series, Series B, Physics, hep-th/9710175; and references therein.

[10] W.-M. Zhang, Phys. Rev. D56, 1528 (1997); E. L. Gubankova and F. Wegner, Phys. Rev. D58, 025012 (1998); B. H. Allen and R. J. Perry, Phys. Rev. D58, 125017 (1998); T. S. Walhout, Phys. Rev. D59, 065011 (1999); c.f. G. P. Lepage, nucl-th/9706029; J. R. Hiller and S. J. Brodsky, Phys. Rev. D59, 016006 (1999); D. G. Robertson, E. S. Swanson, A. P. Szczepaniak, C. R. Ji and S. R. Cotanch, Phys. Rev. D59, 074019 (1999).

[11] M. Brisudová and R.J. Perry, Phys. Rev. D54, 1831 (1996); M. Brisudová, R.J. Perry and K. G. Wilson, Phys. Rev. Lett. 78, 1227 (1997). See also R. J. Perry and K. G. Wilson, Nucl. Phys. B403, 587 (1993) and R. J. Perry, Ann. Phys. 232, 116 (1994).

[12] R. J. Perry, in Hadrons '94, Proceedings, edited by V. Herscovitz and C. Vasconcellos (World Scientific, Singapore, 1995); hep-th/9407056 (revised version); R. J. Perry, in Theory of Hadrons and Light-Front QCD, Proceedings, edited by St. D. Glazek (World Scientific, Singapore, 1995). 
[13] E.g., see, D. E. Soper, in Lattice '96, Proceedings of the International Symposium, St. Louis, Missouri, edited by C. Bernard et al. [Nucl. Phys. B (Proc. Suppl.) 53, 69 (1997)]; M. Göckeler et al., ibid., p. 81, and references therein.

[14] St. D. Głazek, Acta Phys. Pol. B29, 1979 (1998).

[15] St. D. Głazek and K. G. Wilson, Phys. Rev. D57, 3558 (1998).

[16] F. Wegner, Ann. Physik 3, 77 (1994).

[17] A. Mielke, quant-ph/9809086.

[18] K. G. Wilson and St. D. Głazek, in Computational Physics: Proceedings of the Ninth Physics Summer School at the Australian National University, Canberra, Australia, 1996, edited by H. J. Gardner and C. M. Savage (World Scientific, Singapore, 1996).

[19] E.g., see S. Weinberg, The Quantum Theory of Fields (Cambridge University Press, New York, 1995).

[20] B. H. Allen and R. J. Perry, Phys. Rev. D58, 125017 (1998).

[21] R. J. Perry and S. Szpigel, nucl-th/9901079.

[22] R. D. Kylin, B. H. Allen and R. J. Perry, hep-th/9812080.

[23] H. J. Melosh, Phys. Rev. D9, 1095 (1974).

[24] S.-J. Chang, R.G. Root and T.-M. Yan, Phys. Rev. D7, 1133 (1973); S.-J. Chang and T.-M. Yan, ibid. bf 7, 1147 (1973); T.-M. Yan, ibid.7, 1760 (1973); ibid.7, 1780 (1973); and references therein.

[25] S. J. Brodsky, R. Roskies and R. Suaya, Phys. Rev. D8, 4574 (1973).

[26] A. Casher and L. Susskind, Phys. Lett. B44, 171 (1973) and Phys. Rev. D9, 436 (1974); J. Kogut and L. Susskind, Phys. Rep. C8, 75 (1973) and references therein. For a recent presentation, see L. Susskind and M. Burkardt, in Theory of Hadrons and Light-Front QCD, edited by St. D. Głazek (World Scientific, Singapore, 1995); K. G. Wilson and M. Brisudová, ibid.. Refs. [7], [8] and [9] above provide additional discussion.

[27] A. J. Macfarlane and G. Woo, Nucl. Phys. B77, 91 (1974).

[28] J. Collins, Renormalization (Cambridge University Press, Cambridge, 1984).

[29] St. D. Głazek and K. G. Wilson, Phys. Rev. D47, 4657 (1992).

[30] T. Masłowski and M. Więckowski, Phys. Rev. D57, 4976 (1998). 